\title{
Exploration of Language Ecological Niche
}

\author{
Xiaoyan Zeng \\ College of International Education \\ Minzu University of China \\ Beijing, China \\ xiaoyanzeng0313@163.com
}

\author{
Tongtao Zheng \\ Overseas Education College \\ Xiamen University \\ Fujian, China \\ tongtao@xmu.edu.cn
}

\begin{abstract}
Traditionally, the research of teaching Chinese as a foreign language emphasizes classroom teaching more and less emphasizes the learning of outside classroom. In addition to that, second language acquisition theory can't completely explain the effective acquisition of learners under the target language environment or the cultivation of the learners' second language acquisition ability. Based on the beneficial achievement of "ecological niche", this paper puts forward the theory of "language ecological niche" in the perspective of linguistics and applies it to second language acquisition under target language environment, which makes detailed explanation to effectiveness of second language acquisition under target language environment and the cultivation of the learners' second language acquisition ability. This paper also describes the components of language ecological niche in detail and proves that the exploration of language ecological niche contributes to improving efficiency of second language acquisition. 11 students in the elective senior oral Chinese language course are randomly drawn as investigation objects. This paper divides language ecological niches according to language function, sticks to the studentcentered, teacher-guiding, and cultivating students' active learning ability. This research have contributed to understanding the definition and the components of the language ecological niche, the relationship of language ecological niche and second language acquisition, helps to explain how learners acquire language in target language environment from multidiscipline perspective based on the basic language communication system.
\end{abstract}

Keywords-Chinese; language niche; second language acquisition; interaction; active learning ability

\section{INTRODUCTION}

This paper reviews theoretical researches on second language acquisition from the perspectives of linguistics, psychological linguistics, social culture theory and complexity theory. (1) The research on teaching contents from the perspectives of linguistics regards universal grammar as theoretical framework, and it mainly describes and explains learners' second language acquisition statically. "Static state description method is mainly used in the research on second language acquisition from the perspectives of linguistics. That is, many researches only collect language samples of learners respectively, and even some diachronic researches only collect linguistic data separately, which does not establish relationship between language categories and is hard to describe the process of language acquisition.”[1]. (2) The research of psychological linguistics and cognitive linguistics on learners' internal factors mainly focuses on the processing mechanism during second

This research was financially supported project by the Fujian Social Science Planning Project (FJ2017C092) \& Education and Scientific Research Project of Middle and Young Teachers in Fujian (JAS170016). language acquisition process and the elements which influence on this mechanism. Based on abstract theories or special experimental facilities required, concrete operational research can't be implemented by most researchers. The research of recognition mainly measures the language development process of learners in the aspects such as study ability, memory and strategy etc. This research divides complex language cognitive phenomenon into single parameter and does investigation separately. However, the dynamic state of the leaner's recognition process can't be investigated with the interaction. (3) The research of social and cultural theory on the learner's external factors pay more attention to the regulating effect of social culture in second language acquisition and the interactive function of human and society. It also emphasizes the complexity and variability of language acquisition development. Although some theories lay emphasis on dynamic development process of language learning, there are uncontrollable factors in the learning process, such as high cooperation from research objects, time consuming and strong subjectivity of researchers. (4) Complexity theory regards second language acquisition as a complex and dynamic adaptive system. The theory believes that the existence of semanteme is inherent without the communication inherent coding theory. Although complex theories may objectively explain second language acquisition process from macro perspective, they are hard to investigate the efficiency of second language acquisition process in detail from micro perspective or explain how to cultivate second language acquisition ability. Being highly employed in natural science, ecological theory has rarely been used in humanistic and social science. Based on achievement of "ecological niche", this paper puts forward the theory of "language ecological niche" and studies the application of language ecological niche in the micro investigation of second language acquisition process.

\section{DEFINITION TO LANGUAGE ECOLOGICAL NICHE}

With typical affordance, language ecological niche pays more attention to learners with special language learning demand. We believe that language ecological niche highlights the interaction between individual and environment, as well its relationship with individual sensory ability. It specifically involves what things provided by the environment can be used by human. Language ecological niche theory provides new perspectives for the research on how to cultivate learners' second language acquisition ability. It stresses interaction between people, physical environment and social environment. It also emphasizes establishing environmental factor in internal 
and external classroom teaching and cultivating students' active learning ability of perception, acquisition and application of the factors. Learners' second language acquisition ability represents the result of cultivating students' active learning ability.

Individual difference has become the kernel content of second language acquisition research, and language ecological niche makes investigation on complex dynamics of individual difference in second language acquisition. Language ecological niche is the basic presentative unit of language acquisition with multiple characters like dynamics, openness, complexity and adaptation. Under the target language environment, there are different language ecological niches in accordance with different learners. Learners' active learning ability can be acquired through adaptive practice in language ecological niche Chemero thinks, "Animal ecological niche is a kind of environment (situation), and some ability of animals can be practiced under certain environment (situation)" [4].

The improvement of active learning ability can be achieved by cultivating learners' conscious finding and using language ecological niche. Language ecological niche investigates both specific and general characters of all individuals. Language ecological niche is mainly used to investigate individual. It is chaotic at the very start with all kinds of methods and situations, and the verbal communication situations will be confirmed according to contacting frequency. Along with constant intersecting and coinciding of learners' contacting situations, as well as the improvement of contacting frequency. Learners' common characters continually appear clear and become ordered. Language ecological niche can explain the process from chaotic to be ordered. Polechová and Storch to distinguish ecological niche: (1) niche as the description of a species' habitat requirements (surface feature); (2) niche as the ecological function of the species; (3) niche as species position in a community (colony) or the formalization of ecological niche concept [2]. There is similar ecological niche phenomenon of ecology in language through the observation. For detail, ecological niche and language learning can be connected. Paiva thinks ecological niche is an environment regulated by language; ecological niche is a position which induces the usage of language; ecological niche is the function and status of every language user in a utterance community [3].

In a word, language ecological niche refers to the relevant and specific time and space position between learners and different communication objects and the functional relationship and effect between learners and relevant language information resources under the language environment. Learners' active learning ability can be acquire through adaptive practice in a kind of language ecological niche with special demand. There are three elements: Specific time position, Specific space position, Specific function relationship and its effect.

\section{THE COMPONENTS OF LANGUAGE ECOLOGICAL NICHE}

The components of language ecological niche include learner, specific language environment and language interaction. The functional relationship and effect of "four factors" of language ecological niche: the subject of language ecological niche is learners' space-time and dynamic character; specific language environment is the width of language ecological niche; language interaction is an important way of maintaining language ecological niche, Learners' Active Learning Ability Is the Kernel of Language Ecological Niche. Figure 1 shows components of language ecological niche:

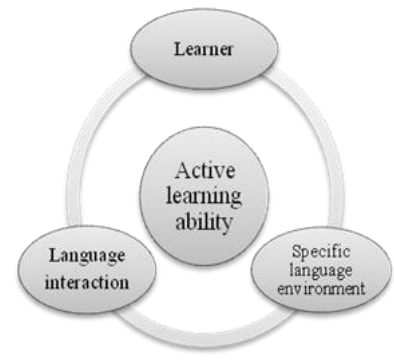

Fig. 1. Three factors constituting language ecological niche

\section{A. Subject of Language Ecological Niche: Learners' Space- Time and Dynamic Characters}

The language ecological niche can't exist without the language user. Learners are not fastened to a certain environment. They constantly change and move. Language ecological niche occupied by every learner has different functions and effects, which provides condition for constituting a complex and ordered language environment. Learners can adjust language ecological niche through learning target language (Chinese) and have specific space-time and dynamic characters.

When learning second language in target language environment, target language environment provides multiple language ecological niches for learners, and these multiple language ecological niches constitute a large language environment. Restaurant, bank, market (supermarket), station and airport are places where learners visit a lot in their daily lives and can be understood as language ecological niches with different situation functions. Because the language ecological niche has close connection with learners, we provide microenvironment for learners' studying and using language according to basic necessities of life. We also take different learning demands of different learners into consideration. Language ecological niche changes constantly for the mobility of learners.

\section{B. Specific Language Environment Is the Width of Language Ecological Niche}

Specific language environment exists as language activity for interaction behavior. However, the modernization has switched the traditional interaction such as the face to face communication. Modern medium likes telephone and network increasingly strengthen occupy the language interaction forms and even break the limits of time and space. Therefore, specific language environment or the language environment without geographical restrictions not only contains language interaction environment of traditional face to face communication, but also the language interaction environment where modern medium like network has been used. This paper mainly focuses on investigating tangible language interaction environment. 
The width of language ecological niche is also called language ecological niche breadth, which is one of quantitative index of language ecological niche characters. Its definition is a learner's ability of using learning resource, the diversification degree of using resource and competition level during learning process. Specific language environment provides learner with learner-centered environment for language interaction according to language environment function. For example, the bank is a specific language environment to learner when he or she handles business in a bank. Specific language environment provides worker, ATM, bulletin board and other customers handling business for the learner. When the learners interact with workers (what kind of business to handle, what certification does he need and how to handle, and so on), communicate with other customers (it might be learning through talk, like asking how to read some words, the words' meaning or how to write something etc., or how to handle a machine), deposit and withdraw money by ATM (complete deposit or withdraw business according to Chinese instruction in the machine). Then there is language ecological niche with banking specific functional relationship and effect.

The wider the language ecological niche of a learner, the lower is his individual specialization degree, which tends to a generalized study. On the contrary, the narrower the language ecological niche of a learner is, the higher is his individual specialization degree, which tends to a generalized study. Generalized learners have a wider language ecological niche. They abandon the usage of resource in narrow range to get in turn the usage of resource in wide range. Specialized learners occupy a narrow language ecological niche. With special adaption ability of using some certain resource, the active learning ability of specialized learners will surpass that of generalized learners when the resource supply is guaranteed and can be created.

\section{Interactive Relationship In Language Ecological Niche}

The interaction in language ecological niche refers to all kinds of interaction relationship generated through language activity. There are two aspects of interaction in language ecological niche: one is the real language interaction between learners and other members; the other is their incorporeal interaction relationship. To learners, even there is no real language interaction behavior, there is internal identification and unified functional language specification, which will maintain all kinds of interaction between learners and factors. Language ecological niche also has availability functional character. Affordance is the interaction relationship between people and their surrounding environment and helps the real semantic network appear and maintain language ecological.

Learners may naturally acquire second language by use of resource provided by environment, and can also establish language ecological niche which may contribute to their learning according to their own requirement (what does the learner do, what he/she wants to do or the function of object, and so on). Language ecological niche mainly refers to learners' active exploring, perceiving and using all kinds of environments when interacting with specific environments. Language learning environment is filled with demand and requirement, chance and restriction, rejection and invitation, as well as permission and constraint.

\section{Learners' Active Learning Ability Is the Kernel of Language Ecological Niche}

According to learners' perception ability and cognitive pattern, each language's ecological niche with specific function relationship and effect is constituted by three factors of learners, specific language environment and interaction. These language environments will be instituted, destructed and recombined endlessly, and finally will show a screw type learning process. To learners, the out-of-class learning in target language environment can better cultivate learners' intercultural communication competence. Based on previous experience, learners' perceptive functions mainly contacts with the outside world directly and enters concerned information through several sense organ galleries like auditory sense, vision and touch sense. Gibson says "to perceive the world is to perceive oneself" [5]. He explains: "the awareness of the world and of one's complementary relations to the world is not separable" [6]. Actually, according to our personal identity, we perceive things according to how did they make connection with us. For instance, one person thinks a cup can be used for storing water, the other might regard the cup as a flower container, but someone else may use it as a container for storing candies. It may happen in language world. When hearing the voice, a musician might appreciate it as a song or beautiful music without seeing the lyrics, or distinguish different musical instruments the sound comes from; a linguist (or a language learner) may be very sensitive to the pronunciation. Learners with different abilities may get together in one language environment but without the same language ecological niche.

\section{SECOND LANGUAGE ACQUISITION INVESTIGATION BASED ON LANGUAGE ECOLOGICAL NICHE}

\section{A. Empirical Analysis}

Second language acquisition is nonlinear learning process with characters of complexity, dynamic nature and adaptability. The aim of investigating second language acquisition based on language ecological niche is to study how to cultivate active learning ability. 11 investigated senior learners of Chinese are randomly selected in this paper, with age between 19 23 and the average age of 20.6 years' old. The teaching material is Developing Chinese Senior Oral Chinese I. This class costs 19 weeks with 76 hours in total, including 9 weeks (36 hours) of experiment. Learners' active learning abilities are investigated through forms of class speech, dynamic evaluation to homework and personal interview. To objectively evaluate their oral ability, comprehensive evaluation will be assessed by two teachers according to evaluation standards. According to language function, investigation contents include language expression of blaming, helpless, attention/requirement, reminding, persuading, warning and criticizing and so on.

There are four standards of evaluation such as: (1) students may properly choose relevant vocabularies and verbal expression; (2) Students can express in Chinese appropriately; (3) students may communicate about broad topics in social life 
and can express their opinions completely and naturally; (4) Students may think like Chinese.

Language ecological niche includes all of the learning activities in and outside classroom within 24 hours. Following teaching methods are adopted to increase students' interaction with environment and improve learning efficiency. (1) Groups can be changed to increase students' interaction. (2) Guiding students to answer questions from easy to difficult. (3) Promoting learn by the frequency, and increasing the interactional information frequency of input and output. Guiding active learning after school refers to teaching tasks, encourage students to occupy language ecological niche related to classroom teaching contents as more as possible, and encourage them to express what they see, what they hear and what they think in oral or written ways. (4) There are two ways to evaluate learning feedback. The first is oral expression (personal speech or free performance); the second is written expression. (5) Comment on students and encourage students' active learning ability.

In the figure, the pretesting result is the result of mid-term examination; the posttest result is the result of final examination. Usual examination includes 50\% extracurricular independent study and $50 \%$ class attendance. The basic requirement of study task of extracurricular independent study is the teaching contents in classroom, which guides students to actively interact with environment and show their independent study condition through written expression or oral expression in class. 11 students have been randomly selected in this paper, and are divided into three types according to their learning contents of Chinese. The first type of students have low class attendance rate and seldom study independently after school (Student Chen and Student Zheng). The second type of students study hard in and outside class, while their independent learning contents have no relationship with class teaching contents (Student Qian and Student Ming). The third type of students study hard in and outside class, and always do independent study after school according to the tasks arranged in classroom (Student Zhang, Student Huang, Student Tian, Student Ou, Student Li, Student Liu and Student Su). Comprehensive assessment results are shown in the following table:

$\approx$ Pretesting result (mid-term examination) $\approx$ Aftertest result (final examination) $\approx$ Regular result

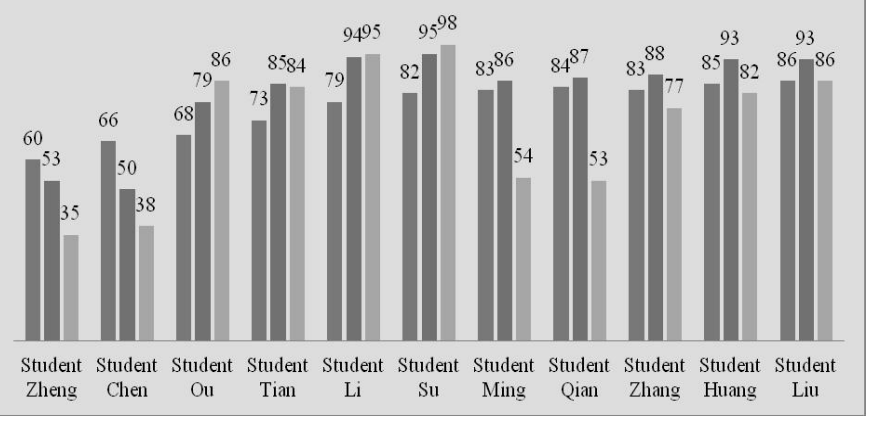

Fig. 2. Comparison table to comprehensive evaluation to 11 randomly selected students
From the figure above we can see, conditions can be subdivided according to students' independent learning and class teaching content based on above classification. Students' using condition to language ecological niche under target language environment is mainly reflected through class attendance rate and independent learning after school. According to students' individual common characters, there are five conditions: the first type students occupy very few language ecological niches relevant to their acquisition language with low attendance rate and no independent study after school. Like Student Zheng and Student Chen with only $70 \%$ attendance rate and few independent study after school. After an interview with Student Chen and Student Zheng, we know that they seldom do activity with classmates and always stay at dormitory or do things by themselves. Under this condition, these students seldom interact with environment, and take few language ecological niche, which is not good for their second language acquisition.

The second type students occupy few language ecological niches relevant to their acquisition language. They have high attendance rate and do independent study after school, while their independent study contents have few relationship with class teaching contents. Like Student Ming and Student Qian with fine Chinese foundation. They learn Chinese mostly in class. Although they interact with environment after school, there is neither pertinence in their learning contents nor defined study goal. They may gain efficient path of learning or rules of acquisition language after several times failure. This condition largely reduces their second language acquisition efficiency in limit time.

The third type students occupy a certain extent of language ecological niches relevant to their acquisition language. They have high attendance rate. Although they study after school, their study contents have few connection with class teaching. Like Student Zhang, who is good at learning in and out-ofclass time with better learning foundation of Chinese, however, his Chinese does not improve significantly because of his lower relevance of independent learning with class teaching.

The fourth type students occupy many language ecological niches relevant to their acquisition language. They have high attendance rate and study the contents which have high relevance with class teaching contents after school, such as Student Huang and Student Liu with fine Chinese foundation. On the contrary, though Student $\mathrm{Ou}$ and Student Tian have weak Chinese foundation, these four students all get an significant improvement. Their improving range from aftertest to pretesting is 7-12 scores, with a more than middle average usual score (among 82-86). This shows that, no matter the students with good or bad Chinese at the beginning, their examination results improve obviously under teachers' guidance in class and by cultivating their active learning ability in out-of-class time.

The fifth type students have a large number of language ecological niches relevant to their acquisition language. These students have high attendance rate, study after school actively, pay attention to the combination of independent learning with class teaching, and will apply to what they have learned in social practice or social survey except for finishing previewing 
and reviewing of teaching materials. Such as Student Li and Student Su who get obvious improvement, ranking first and second with scores of 94 and 95. They also have excellent regular scores of 95 and 98. After an interview we know that, under teachers' guidance, these two students participate in all kinds of activities actively after school, and actively establish Chinese learning environment. For example, they will take the initiative to talk with unacquainted Chinese students when having dinners in canteen. They will join parties of their Chinese friends and interact with environments constantly. In interaction process, they apply what they have learned in class or from teaching materials into practical communication environment. From their written or oral feedback we can see what they learned contents of Chinese, we will observe and think about what they saw or heard. If the have some questions, they will consult with friends, search the internet or interact with teachers or students to analyze and solve their puzzled questions. They will record teachers' written or oral comments and make progress during continuous correction. They insist learning Chinese for 10 hours every day, among which, 4-7 hours are used to learn things relevant to class teaching contents. The result increase rate of distribution condition of 11 students is shown in the following figure 2 .

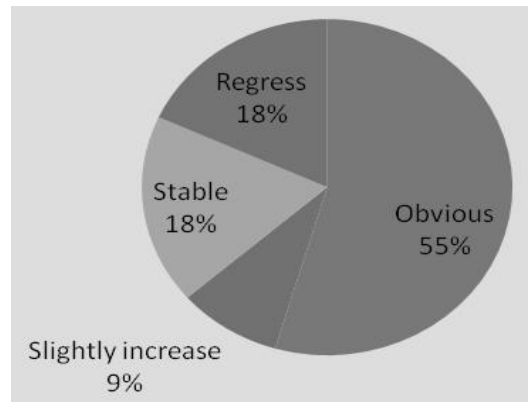

Fig. 3. Result increase rate distribution figure

From the figure 2 we can see that, there is a rising trend in overall results, which occupies $82 \%$. $55 \%$ of the results improve obviously. $18 \%$ of the results show regression trend. The main reason of regressing is the two students' low attendance in class and social activity, and there is no improvement in their results. This shows, on the basis of guaranteeing class teaching efficiency, teachers should also pay attention to cultivate students' active learning ability after school to improve their Chinese acquisition efficiency in a limited time, which is to improve Chinese acquisition efficiency by focusing on specialized learning.

In a word, these five types of students reflect the effect of language ecological niche in improving students' language acquisition in different degrees. The broad language ecological niches of the first and second type students tend to be a generalized learning. Generalized learners have a broad language ecological niche. They use resources in a broad range through abandoning the resources in a narrow range. However, the narrow language ecological niches of third, fourth and fifth students tend to be specialized learners. Specialized learners occupy a narrow language ecological niche. With special adaption ability of using some certain resource, the active learning ability of specialized learners will surpass that of generalized learners when the resource supply is guaranteed and can be created.

\section{B. Contact Occasions and Frequency in Language Communication}

According to language function, namely language expression of blaming, helpless, attention/requirement, reminding, persuading, warning and criticizing and so on. We get the statistics of the contact occasions and frequency of learners in the time of outside classroom, based on individual interview and homework feedback as shown below.

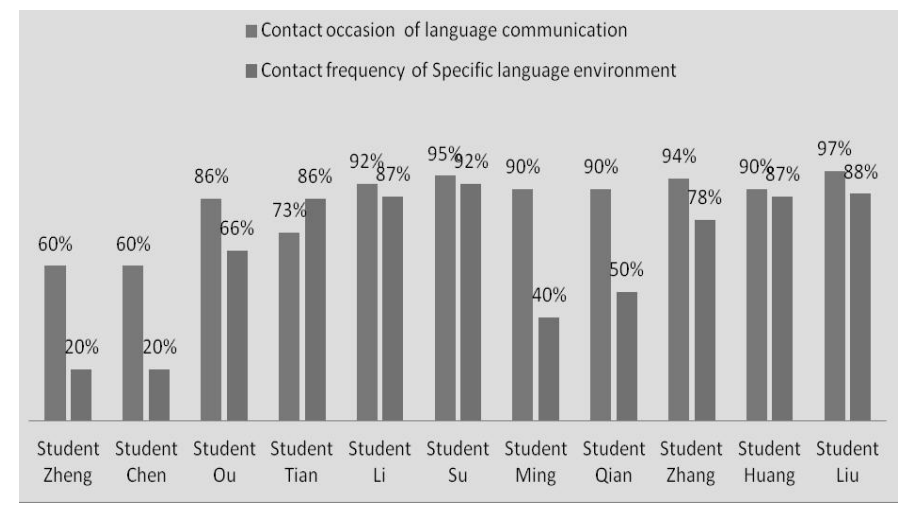

Fig. 4. Learners' individual contact occasions and frequency in language communication

Contact occasions or mode of language communication provides basic condition for contact frequency of language communication. Contact frequency of language communication is the occurrence numbers of semantic unit with communication function in society. Learners input and output semantic units with communication function in different environment. When these semantic units with communication function are repeated for many times, the high and low frequency appeared in contact. For example, a French overseas student once shared his experience:

There is large difference in Chinese and French traffic situation, which is mainly reflected in Chinese and French traffic rules. Compared with China, France has more perfect traffic rules and low traffic accident rate. In addition, France has good quality building materials with long service life rather than China. Because of imperfect traffic rules and Chinese' driving without abiding by traffic rules strictly, China has high traffic accident rate. I have experienced a traffic accident. My friend and I drive on the expressway regularly according to traffic rule. A car behind us surpassed us from right low speed lane, while he should have surpassed us from left fast speed lane. He ran into a private car Which was present at the overtaking lane. There was no any danger reminding mark to this private car. Meanwhile, our car was also knocked. I lose my friend in that traffic accident and felt terribly grieved. I hope China's traffic rules will be perfected at an early day, and wish all of us could cherish our life and abide by traffic rules. 
Student Liu occupies the space and the time of language ecological niche. There is expressway, hospital, home and classroom in the space language ecological niche. Target language environment constantly repeats these semantic units with communication function for Student Liu and provides him diversified communication occasions. The factor of time connects independent language ecological niches like expressway, hospital and classroom into a dynamic language learning environment. Different communication occasions provide different communication objects for learners. For example, there are policemen in expressway to deal with car accidents; doctors, nurses and other patients in hospital; friends and relatives at home; teachers' guidance and classmates' interaction in the classroom. The learner describes the details about the accident, his sorrow and opinions, and so on. The topic triggers learner's Recalling and thinking. In addition, the learner continually improves his active learning ability through writing diaries, solving problems through searching information about traffic in the internet, or publishing daily records, for example Facebook, Twitter and so on.

The narrower of Student Liu's language ecological niche is, the higher his specialized degree. With special adaption ability of using some certain resource, the active learning ability of specialized learners will surpass that of generalized learners when the resource supply is guaranteed and can be created. In addition, target language countries provide learners contact frequency of language communication. The important reasons of increasing language contact frequency may focus on their specific elements such as time sequence, position change, communicational diversity and affection. Learners perceive differences of cultures, think through comparison and actively put forward, analyze and solve problems.

With more contact with traffic words, Liu may express this topic in Chinese in a more complex, fluent and accurate way than the topic of 'comparison between Chinese and French education'. A narrow language ecological niche and high priority degree of acquisition target language can be achieved through high contact and interaction frequency, which tends to be a specialized learning. This case fully shows that the active learning ability of specialized learners is better than that of generalized learners.

In a word, communication contact occasions and frequency are key factors for second language acquisition under target language environment, and directly related to the development of learners' active learning ability. Comparing language ecological niche with traditional context view, language ecological niche is more suitable to explain people's language acquisition ability. The starting point of research on language ecological niche is to have comprehensive observation and description of language acquisition under real environment. It emphasizes multi-angle analysis of the disciplines of second language acquisition and its development during the interaction of learners and environments. As a dynamic process of communicators' fusing and exploring, language ecological niche may explain and develop learners' language communication ability. It is completely in conformity with human acquisition process to regard learners' acquisition process as a series of dynamic time and space, and then observe learners' language acquisition. This is also the largest advantage of language ecological niche. That is to say, learners acquire language ability in different time and space. Different active learning ability and passive learning ability will directly result in different language acquisition.

\section{SUMMARY}

This paper mainly explores the language ecological niche in the process of second language acquisition. Class teaching could not offer all the language ecological niches for language development and can't support learners view in a broader range. Learners' ability, what they want to do (because of active demand or being forced by ambient pressure), the function and quantity of objects are all closely connected to learners and will help specific learners to create language ecological niche. The kernel of language ecological niches can be concluded into following three aspects: (I) Language ecological niche is the environment which can be adjusted by learners through target language (Chinese). Language ecological niche helps language acquisition learners cultivate active learning ability. (II) Language ecological niche provides diverse language environments for language interaction which can meet learners' special requirement. Learners' active interaction with environments will help them gain more language information and resources and promote their language acquisition efficiency. (III) Language ecological niche has influence and position for learners with special language acquisition demand, and provides guarantee for learners' ordered adaptive acquisition. (IV) Language ecological niche describes learners' language acquisition environment and dynamic acquisition process. This paper explains second language acquisition procedure from multidiscipline perspective, investigates second language acquisition in language ecological niche based on the basic supportive system in language communication, and has important significance to widen view of second language research.

\section{REFERENCES}

[1] Wang, Lulu, Dai Weidong. Review to Second Language Acquisition Research Method. Foreign Language World, no. 5, 2014, pp. 29-37.

[2] Polechová, J., \& Storch, D. Ecological niche. In S.E. Jorgensen \& B. Fath(Eds.).Encyclopedia of ecology. Oxford: Elsevier, 2008, pp. 10881097.

[3] Paiva, V. L. M.O. Affordance beyond the classroom. In P. Benson \& H. Reinders(Eds.), Beyond the classroom: The theory and practice of informal language learning and teaching. Palgrave: Macmillan, 2001,pp. 59-57.

[4] Chemero, A. 2003. An Outline of a Theory of Affordances. Ecological Psychology, vol. 15, 2003, pp. 181-195.

[5] Gibson,J.J. The Ecological Approach to Visual Perception. Boston: Houghton Mifflin Company, 1979, pp. xiii.

[6] Gibson, J.J. The Ecological Approach to Visual Perception. Hillsdale, New Jersey: Lawrence Erlbaum, 1986, pp.129-141.

[7] Michael Hornsby. Fingding an ideological niche for new speakers in language community. Language, Culture and Curriculum, vol.30, no.1, 2017, pp.91-104.

[8] Tommaso Bertolotti \& Lorenzo Magnani. Theoretical considerations on cognitive niche construction. Synthese, vol. 194, 2017, 4757-4779. 